\title{
Localized Angiosarcoma, Not One Disease: A Retrospective Single-Center Study on Prognosis Depending on the Primary Site and Etiology
}

\author{
Inna Schott, ${ }^{1}$ Sven-Thorsten Liffers, ${ }^{2,3}$ Farhad Farzaliyev $\left(\mathbb{D},{ }^{4}\right.$ Johanna Falkenhorst $\mathbb{D}^{1,5}$ \\ Hans-Ulrich Steinau, ${ }^{6}$ Jürgen-Walter Treckmann $\left(\mathbb{D},{ }^{6}\right.$ Lars Erik Podleska ${ }^{(D)}{ }^{4}$ \\ Christoph Pöttgen (D), ${ }^{7}$ Hans-Ulrich Schildhaus (D), ${ }^{8}$ Marit Ahrens (D), ${ }^{9}$ Uta Dirksen (D), ${ }^{10,5}$ \\ Fatma-Zehra Murat $\left(\mathbb{D},{ }^{1}\right.$ Jens T. Siveke $\left(\mathbb{D},{ }^{1,2,3}\right.$ Sebastian Bauer ${ }^{1 D},{ }^{1,5}$ \\ and Rainer Hamacher iD $^{1,5}$ \\ ${ }^{1}$ Department of Medical Oncology, Sarcoma Center, West German Cancer Center, University Hospital Essen, Essen, Germany \\ ${ }^{2}$ Bridge Institute of Experimental Tumor Therapy, West German Cancer Center, University Hospital Essen, Essen, Germany \\ ${ }^{3}$ Division of Solid Tumor Translational Oncology, \\ German Cancer Consortium (DKTK,Partner Site Essen) and German Cancer Research Center, DKFZ, Heidelberg, Germany \\ ${ }^{4}$ Department of Orthopedic Oncology, West German Cancer Center, University Hospital Essen, \\ Essen, Germany \\ ${ }^{5}$ German Cancer Consortium (DKTK), Partner Site University Hospital Essen, Essen, Germany \\ ${ }^{6}$ Department of General, Visceral and Transplantion Surgery, Sarcoma Center, West German Cancer Center, \\ University Hospital Essen, Essen, Germany \\ ${ }^{7}$ Department of Radiotherapy, West German Cancer Center, University Hospital Essen, Essen, Germany \\ ${ }^{8}$ Institute of Pathology, West German Cancer Center, University Hospital Essen, \\ Essen, Germany \\ ${ }^{9}$ Medical Clinic II, University Hospital Frankfurt, Frankfurt am Main, Germany \\ ${ }^{10}$ Pediatrics III Pediatric Hematology, Oncology, Immunology, Cardiology, Pulmonology, West German Cancer Center, \\ University Hospital Essen, Essen, Germany
}

Correspondence should be addressed to Rainer Hamacher; rainer.hamacher@uk-essen.de

Received 24 March 2021; Revised 16 June 2021; Accepted 14 August 2021; Published 11 September 2021

Academic Editor: Peter C. Ferguson

Copyright ( 92021 Inna Schott et al. This is an open access article distributed under the Creative Commons Attribution License, which permits unrestricted use, distribution, and reproduction in any medium, provided the original work is properly cited.

Background. Angiosarcomas are rare and heterogeneous tumors with poor prognosis. The clinical subtypes are classified depending on the primary site and etiology. Methods. We conducted a retrospective, monocentric study of 136 patients with localized AS between May 1985 and November 2018. Overall survival (OS), local recurrence-free survival (LRFS), and metastasis-free survival (MFS) were estimated using the Kaplan-Meier method. To identify prognostic factors, univariate and multivariate analyses were performed based on Cox regressions. Results. The median age was 67 years (19-72.8 years). Primary sites were cutaneous $(27.2 \%)$, breast $(38.2 \%)$, and deep soft tissue $(34.6 \%)$. The majority was primary angiosarcomas (55.9\%) followed by postradiation (40.4\%) and chronic lymphedema angiosarcomas (2.9\%). Prognosis significantly differed depending on the primary site and etiology. Shortest median OS and MFS were observed in deep soft tissue angiosarcomas, whereas cutaneous angiosarcomas, angiosarcomas of the breast, and radiation-associated angiosarcomas displayed worse median LRFS. Univariate analyses showed better OS for tumor size $<10 \mathrm{~cm}(p=0.009)$, negative surgical margins $(p=0.021)$, and negative lymph node status $(p=0.007)$. LRFS and MFS were longer for tumor size $<10 \mathrm{~cm}(p=0.012$ and $p=0.013)$. In multivariate analyses, age $<70$ years was the only independent positive prognostic factor for OS in all subgroups. For LRFS, secondary AS of the breast was a negative prognostic factor (HR: 2.35; $p=0.035$ ). Conclusions. Different behaviors and prognoses depending on the primary site and etiology should be considered for the treatment of this heterogeneous disease. In cutaneous angiosarcomas of the head/neck and postradiation angiosarcomas of the breast, local recurrence seems to have a crucial impact on OS. 
Therefore, improved local therapies and local tumor staging may have to be implemented. However, in deep soft tissue angiosarcomas, distant recurrence seems to have a major influence on prognosis, which indicates a benefit of additional perioperative chemotherapy.

\section{Introduction}

Angiosarcomas are very rare and malignant tumors that share markers of differentiation with endothelial cells of blood or lymphatic vessels [1]. These tumors account for only $2-5 \%$ of soft tissue sarcomas with an incidence of only 1.5 to 2.8 per million per year [1-5]. Angiosarcomas occur everywhere in the body as primary tumors or secondary tumors related to radiation therapy or chronic lymphedema. Depending on the anatomic location and etiology, they are typically subdivided into cutaneous angiosarcomas, mainly on the head and neck, radiation-induced angiosarcomas, mainly of the breast, lymphedema-associated angiosarcomas, primary breast angiosarcomas, and soft tissue angiosarcomas [1]. The prognosis of angiosarcomas is generally poor with median overall survival reported between 30 and 50 months and 5year overall survival rates between $10 \%$ and $50 \%$, while localized angiosarcomas range around $40 \%$, and metastasized disease drops to around $15 \%[1,5-8]$. The impact of the type of angiosarcoma on prognosis is not well studied, and particularly, generic staging classifications appear to have limited value in angiosarcomas. However, an increasing number of studies demonstrate distinct biological and clinical differences between certain subtypes. Cutaneous angiosarcomas of the head and neck have been described to be associated with high tumor mutational burden (TMB) and dominant ultraviolet damage mutational signature which makes them particular candidates for immune checkpoint therapy [9-11]. Radiationand lymphedema-associated angiosarcomas were associated with MYC gene amplification, whereas primary angiosarcomas of the breast showed a high rate of PIK3CA-activating mutations [11, 12]. Regarding the treatment of localized angiosarcomas, randomized clinical trials do not exist. Few studies investigated the relevance of perioperative treatment with radiation therapy and chemotherapy. However, general recommendation cannot not be drawn yet $[1,13-16]$. In this context, angiosarcomas are treated according to other high-grade sarcomas with the primary aim of complete resection with wide margins [1].

In this retrospective single-center study, we systematically addressed the value of several factors in localized angiosarcomas with focus on the main clinical subtypes according to the anatomic site and etiology. Our study demonstrates a distinct different behavior of the subtypes with regard to local recurrence, metastasis, and overall survival. While deep soft tissue angiosarcomas showed a high risk of metastasis, in cutaneous angiosarcomas, angiosarcomas of the breast, and secondary angiosarcomas, the risk of local recurrence was increased. Risk factors for reduced survival were tumor size $>10 \mathrm{~cm}$, positive surgical margins and positive or unknown lymph node status, and age $>70$ years.

\section{Materials and Methods}

2.1. Study Design and Population. Patients with the diagnosis of angiosarcoma between May 1st, 1985, and November 30th, 2018, were identified using the institutional tumor documentation database of the West German Cancer Center, University Hospital Essen, Essen, Germany. All consecutive patients with a pathological diagnosis of angiosarcoma were entered in the electronic database. Angiosarcoma was defined using the International Classification of Diseases for Oncology, 3rd edition (ICD-O-3), morphological code 9120/3. Age at diagnosis, sex, histology, etiology, tumor size, tumor location, nodal status, occurrence and date of local relapse, occurrence, location, and date of metastasis, surgery (extent of resection: R0, R1, or R2), perioperative treatment, and date of death/last encounter were collected for each patient by the retrospective chart review. Angiosarcoma patients were classified based on etiology (spontaneous, radiation-, and lymphedema-associated) and anatomic location (cutaneous, breast/trunk, and deep tissue including patients with angiosarcoma of organs). Time intervals for the analysis were calculated from the date of diagnosis.

2.2. Statistical Considerations. Descriptive statistical analysis was used. Therefore, frequency of occurrence and percentage were calculated for each of the independent variables. Overall survival (OS), local recurrence-free survival (LRFS), metastasis-free survival (MFS), and survival rates (2- and 5-year) were calculated using the Kaplan-Meier method and survival tables. Differences in OS, LRFS, and MFS were determined using the log-rank test. To identify prognostic factors for survival, univariable analyses were performed using Cox regression analysis. On the basis of clinical relevance and significance in univariable tests, factors were selected for multivariate Cox regression analysis. Statistical analyses were performed with IBM SPSS Statistics version 27 (Armonk, NY, USA) and the R survival package (Therneau T (2020); a package for survival analysis in R; version 3.2.7, http://www.rproject.org) using R (version 3.6.3, http://www.r-project.org).

\section{Results}

3.1. Demographics and Disease Characteristics. The demographic, tumor, and treatment characteristics are presented in Table 1. We identified 136 patients with localized disease (UICC II-IIIB) at the time of diagnosis with a slight dominance of female patients. The majority of patients was younger than 70 years at diagnosis. Most patients presented with spontaneous angiosarcomas, followed by radiation-associated angiosarcomas and only four with lymphedema-associated angiosarcoma, so called Stewart-Treves syndrome. The primary location was the 
TABLe 1: Patient characteristics for the cohort of localized angiosarcomas.

\begin{tabular}{|c|c|c|c|}
\hline & & $N$ & $\%$ \\
\hline Follow-up time & Median follow-up time: 19.1 months (0.1-428.0 months) & 136 & \\
\hline \multirow{3}{*}{ Age } & Median age: 67 years (19-72.8 years) & 136 & \\
\hline & $<70$ years & $78 / 136$ & 57.4 \\
\hline & $\geq 70$ years & $58 / 136$ & 42.6 \\
\hline \multirow{2}{*}{ Sex } & Female & $81 / 136$ & 59.6 \\
\hline & Male & $55 / 136$ & 40.4 \\
\hline \multirow{4}{*}{ Tumor size } & $\leq 5 \mathrm{~cm}$ & $50 / 136$ & 36.8 \\
\hline & $>5 \mathrm{~cm}$ & $19 / 136$ & 14.0 \\
\hline & $\geq 10 \mathrm{~cm}$ & $21 / 136$ & 16.2 \\
\hline & Unknown & $45 / 136$ & 33.1 \\
\hline \multirow{4}{*}{ Etiology } & Primary & $76 / 136$ & 55.9 \\
\hline & Postradiation & $55 / 136$ & 40.4 \\
\hline & Chronic lymphedema & $4 / 136$ & 2.9 \\
\hline & Unknown & $1 / 136$ & 0.7 \\
\hline \multirow{9}{*}{ Localization } & Cutaneous & $37 / 136$ & 27.2 \\
\hline & (i) Head and neck & $25 / 136$ & 18.4 \\
\hline & (ii) Others & $12 / 136$ & 8.8 \\
\hline & Breast & $52 / 136$ & 38.2 \\
\hline & Others (deep tissue) & $47 / 136$ & 34.6 \\
\hline & (i) Abdomen & $15 / 136$ & 11.0 \\
\hline & (ii) Thorax & $9 / 136$ & 6.6 \\
\hline & (iii) Limbs & $16 / 136$ & 11.8 \\
\hline & (iv) Others & $7 / 136$ & 5.1 \\
\hline \multirow{3}{*}{ Lymph node } & N0 & $87 / 136$ & 64.0 \\
\hline & N1 & $8 / 136$ & 5.9 \\
\hline & $\mathrm{Nx}$ & $41 / 136$ & 30.1 \\
\hline \multirow{5}{*}{ Margin status } & R0 & $65 / 136$ & 47.8 \\
\hline & $\mathrm{R} 1$ & $26 / 136$ & 19.1 \\
\hline & $\mathrm{R} 2$ & $4 / 136$ & 2.9 \\
\hline & $\mathrm{Rx}$ & $23 / 136$ & 16.9 \\
\hline & No surgery & $18 / 136$ & 13.2 \\
\hline \multirow{2}{*}{ Treatment } & Only surgery & $69 / 119$ & 58.0 \\
\hline & Perioperative therapy & $50 / 119$ & 42.0 \\
\hline \multirow{4}{*}{ Metachronous metastasis } & All & $45 / 136$ & 33.1 \\
\hline & Cutaneous & $12 / 136$ & 8.8 \\
\hline & Breast & $15 / 136$ & 11.0 \\
\hline & Others (deep tissue) & $19 / 136$ & 14.0 \\
\hline
\end{tabular}

breast in 52 patients (38.2\%) and other deep tissues in 47 patients $(34.6 \%)$, and 37 patients were with cutaneous angiosarcoma $(27.2 \%)$. For the majority of patients, the tumor size was $\leq 5 \mathrm{~cm}$. The distribution of tumors $>5 \mathrm{~cm}$ and $\geq 10 \mathrm{~cm}$ was similar. However, in a significant number of patients, the tumor size was not known. For 119 patients, comprehensive data on treatment were available, where in 69 patients $(58.0 \%)$, only surgery was performed and 50 patients (42\%) received perioperative treatment. Most patients presented with a negative lymph node status, and R0 resection was achieved in $47.8 \%$. However, in a significant number of patients, the initial lymph node status and margin status could not be determined. Metachronous metastasis was observed in 45 patients (33.1\%).

3.2. Prognostic Factors for Overall Survival. The 2-year and 5year overall survival rates for the entire cohort of localized angiosarcoma were $54 \%$ and $30 \%$, respectively. The median overall survival was 31.8 months. Kaplan-Meier curves showed a significant reduced median overall survival for deep soft tissue angiosarcomas compared to cutaneous angiosarcomas and angiosarcomas of the breast (Figure 1(a)). Hereby, angiosarcomas of the breast, which were mainly radiation-associated, also showed a better 5-year survival rate with $38 \%$ compared to cutaneous angiosarcomas with $21 \%$ and deep soft tissue angiosarcomas with 25\% (Figure 1(a)). A slightly better outcome was demonstrated in radiation-associated versus spontaneous angiosarcomas, although not statistically significant (Figure 1(b)). The four patients with lymphedema-associated angiosarcoma showed the shortest median overall survival, and all died during follow-up time (Figure 1(b)). Univariate analyses for parameters of tumor staging showed a significantly reduced median overall survival for tumor size $\geq 10 \mathrm{~cm}$, positive or unknown lymph mode status, and positive surgical margins (Table 2).

3.3. Prognostic Factors for Local Recurrence-Free Survival. Next, we analyzed the factors that determine the risk of local relapse. The entire cohort of localized angiosarcomas 


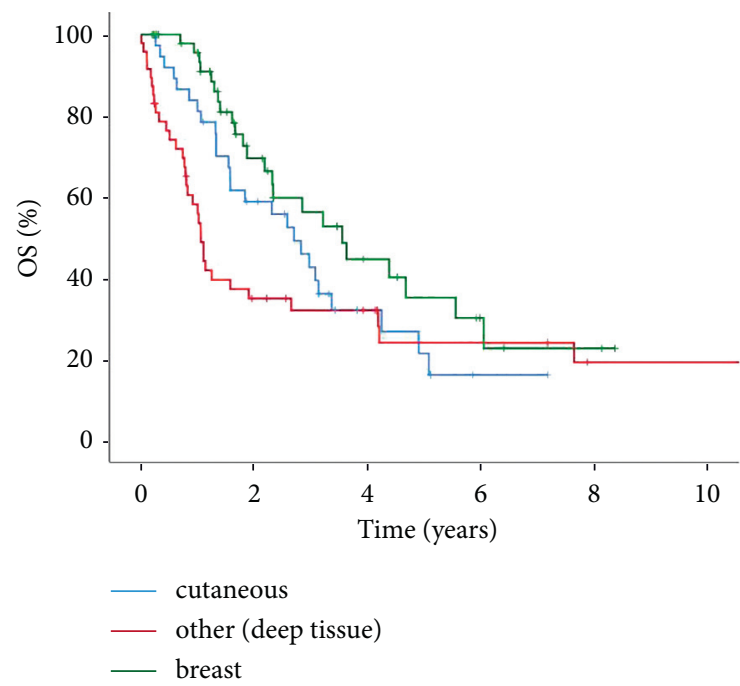

(a)

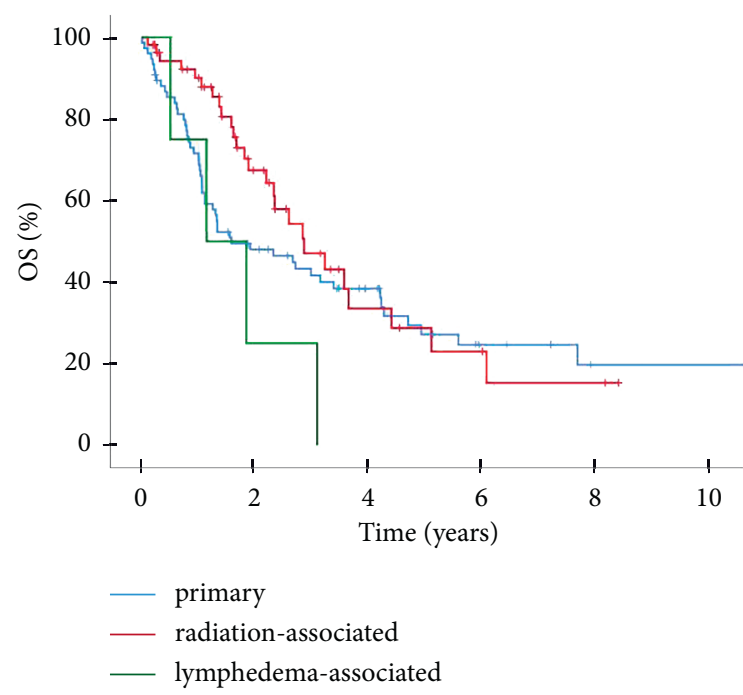

(b)

Figure 1: Overall survival (OS). Kaplan-Meier curves showing 10-year OS (a) comparing deep soft tissue angiosarcomas ( $N=47$ ), cutaneous angiosarcomas $(N=37)$, and angiosarcomas of the breast $(N=52)$ with a median OS of 12.7 vs. $35.6(p=0.213)$ and 42.6 months $\left(p=0.006^{* *}\right)$ and $(b)$ comparing primary $(N=76)$, radiation-associated $(N=55)$, and lymphedema-associated $(N=4)$ angiosarcomas with a median OS of $18.9,34.2$, and 13.6 months $(p>0.05)$.

TABLE 2: Univariate analysis for median overall survival, local recurrence-free survival, and metastasis-free survival displayed in months and $p$ value.

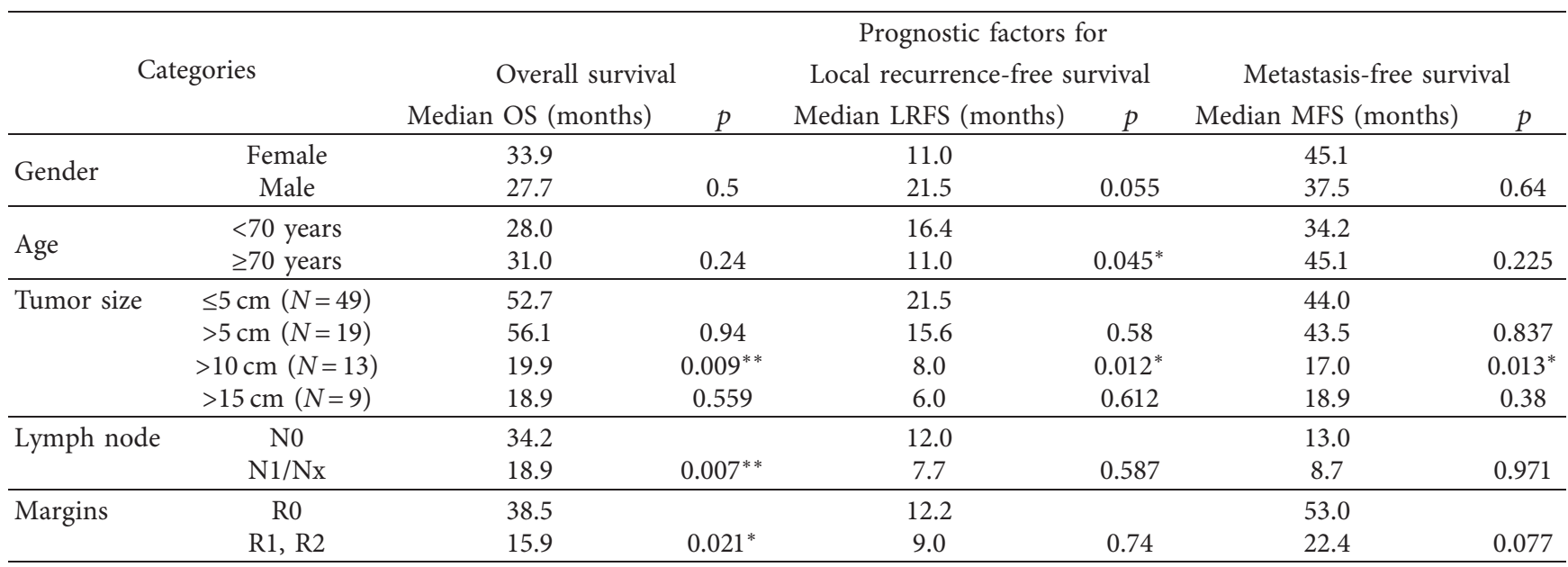

${ }^{*} p \leq 0.05 ;{ }^{* *} p \leq 0.01 ;{ }^{* * *} p \leq 0.001$.

showed a 2-year and 5-year local recurrence-free rates of $22 \%$ and $14 \%$, respectively. The median local recurrencefree survival was 12.2 months. For deep soft tissue angiosarcomas, the median local recurrence-free survival was significantly longer compared to cutaneous angiosarcomas and angiosarcomas of the breast (Figure 2(a)). Regarding the etiology, primary angiosarcomas showed significantly longer local recurrence-free survival compared to radiation-associated angiosarcomas (Figure 2(b)). The four patients with lymphedema-associated angiosarcoma had shortest local recurrence-free survival (Figure 2(b)). Additionally, univariate analysis revealed that tumor size $>10 \mathrm{~cm}$ was associated with a significantly reduced local recurrence-free survival in the entire cohort of localized angiosarcomas (Table 2). Due to the small number, the subentities were excluded for further analysis. Moreover, patients $\geq 70$ years showed a reduced local recurrence-free survival (Table 2 ).

3.4. Prognostic Factors for Metastasis-Free Survival. The median metastasis-free survival for the cohort of localized angiosarcomas was 44.0 months. Similar to the analysis for overall survival, deep soft tissue angiosarcomas showed a reduced median metastasis-free survival compared to cutaneous angiosarcomas and angiosarcomas of the breast, at 


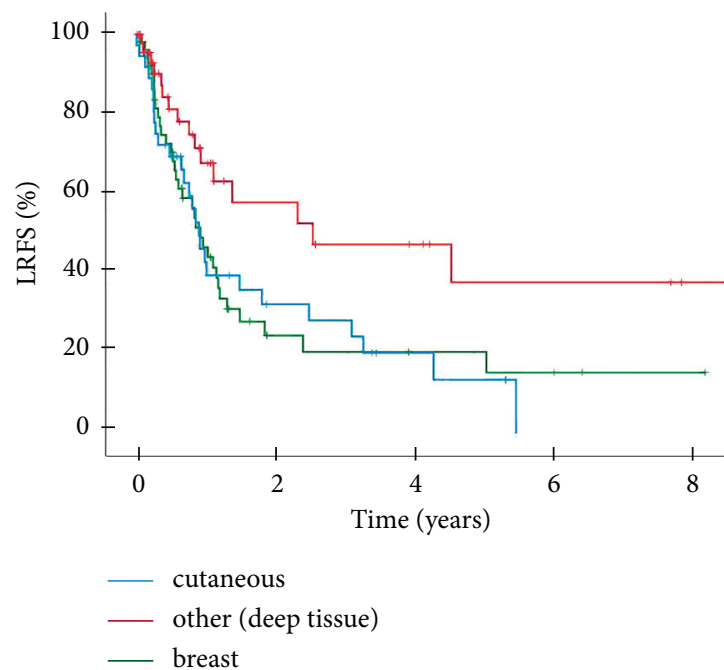

(a)

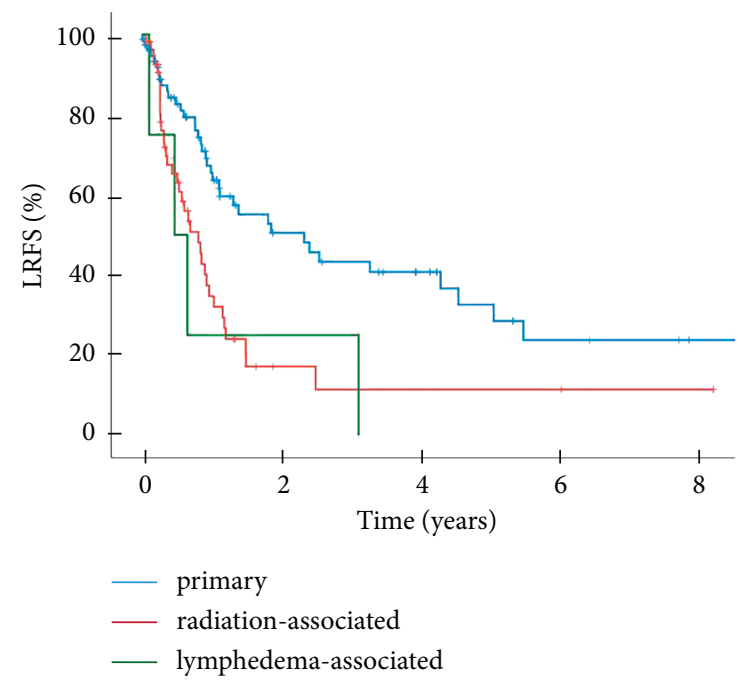

(b)

FIGURE 2: Local recurrence-free survival (LRFS). Kaplan-Meier curves showing 8-year LRFS (a) comparing deep soft tissue angiosarcomas $(N=47)$, cutaneous angiosarcomas $(N=37)$, and angiosarcomas of the breast $(N=52)$ with a median LRFS of 30.2 vs. 10.7 vs. 11.0 months $\left(p=0.013^{*} ; p=0.018^{*}\right)$ and (b) comparing primary $(N=77)$, radiation-associated $(N=45)$, and lymphedemaassociated $(N=4)$ angiosarcomas with a median LRFS of 27.6 vs. 9.6 vs. 5.5 months $\left(p=0.001^{* * *} ; p=\right.$ n.a. $)$.

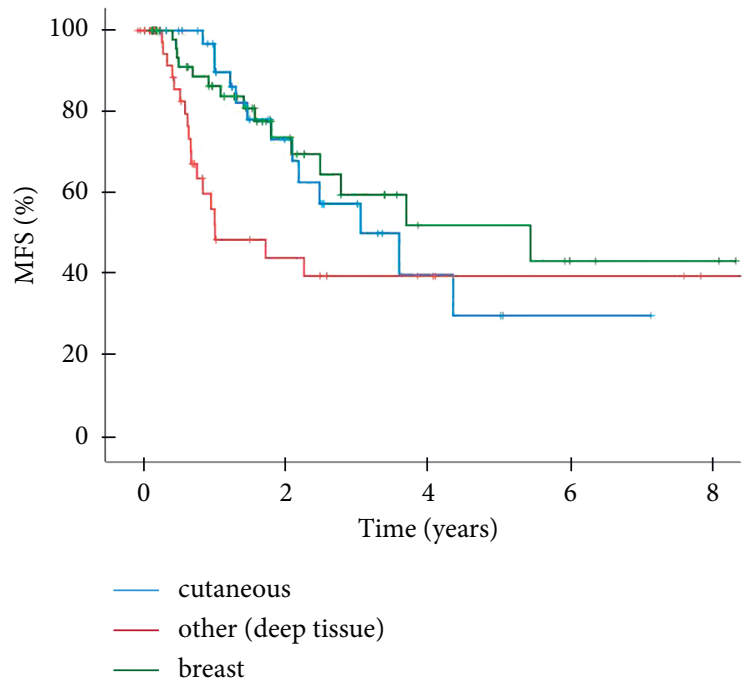

(a)

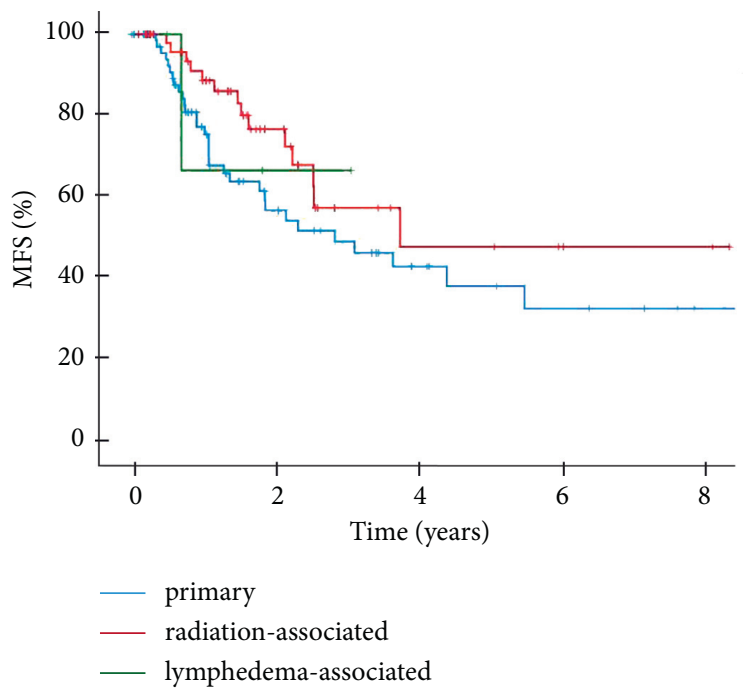

(b)

Figure 3: Metastasis-free survival (MFS). Kaplan-Meier curves showing 8-years MFS (a) comparing deep soft tissue angiosarcomas ( $N=46$ ), cutaneous angiosarcomas $(N=37)$, and angiosarcomas of the breast $(N=52)$ with a median MFS of 13.0 vs. 44.0 vs. 66.0 months ( $p=0.068$; $\left.p=0.034^{*}\right)$ and $(b)$ comparing primary $(N=77)$ and radiation-associated $(N=55)$ angiosarcomas with a median MFS of $34.2 \mathrm{vs.} 45.1(p=0.103)$.

which was significant to the latter (Figure 3(a)). The difference in metastasis-free survival between primary and radiation-associated angiosarcomas was not significant (Figure 3(b)). Only one patient with lymphedema-associated angiosarcoma showed distant metastases to the thigh and femoral head at 8.4 months after diagnosis. Similar to the previous analysis, tumor size $>10 \mathrm{~cm}$ is associated with a significantly lower median metastasis-free survival (Table 2).
3.5. Multivariate Analyses. To identify independent prognostic factors, we performed multivariate Cox regression analyses on overall, local recurrence-free, and metastasis-free survival. The only significant prognostic factor for better overall survival in all subgroups was age $<70$ years (Figure 4 and Supplement Figure 1). The primary site had no significant impact on survival in multivariate analyses. However, similar to Kaplan-Meier analyses, prognosis for overall survival was in disfavor of deep soft tissue angiosarcomas (Figure 4(a)). 


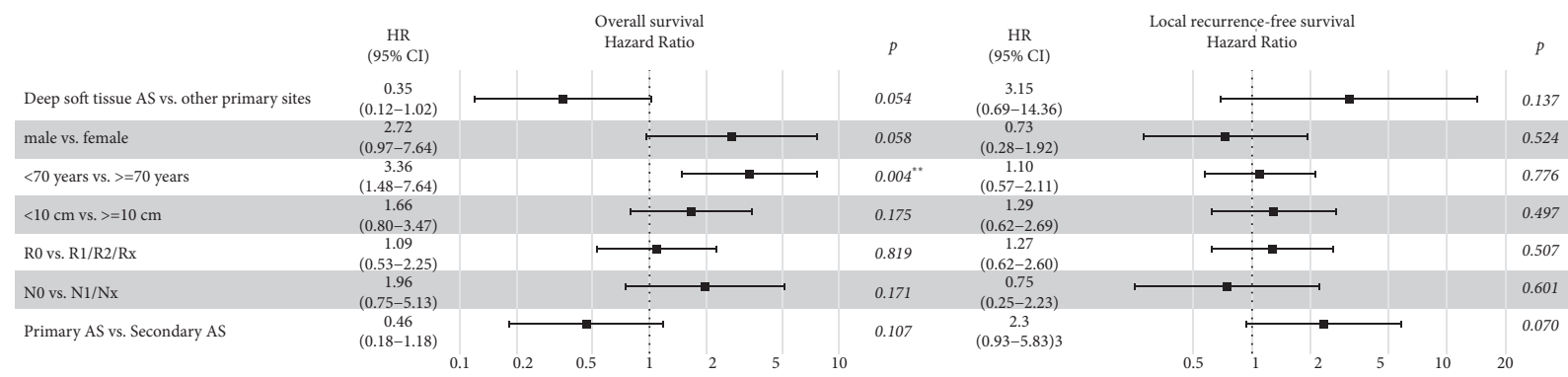

(a)

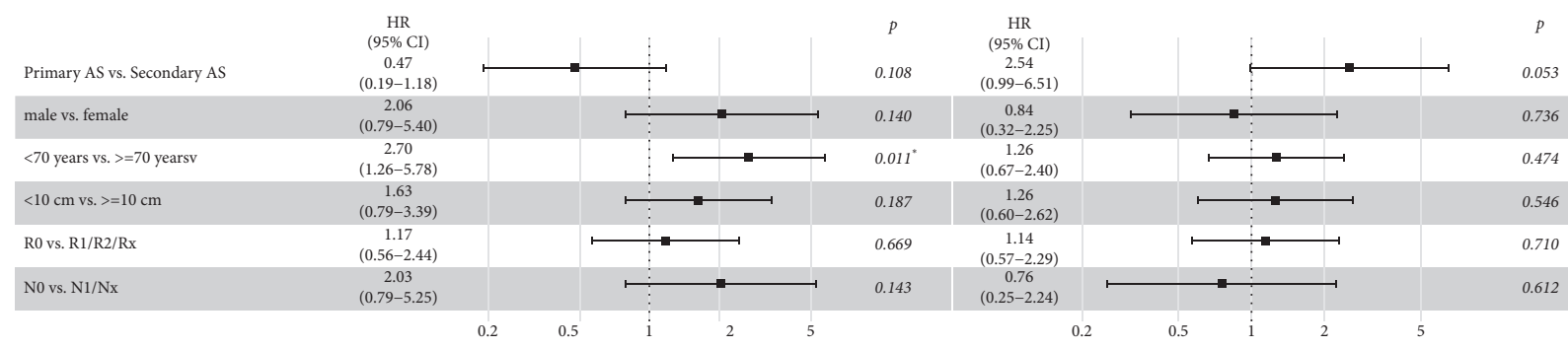

(b)

Figure 4: Multivariate analysis. Forest plots of multivariate Cox regression analysis on overall survival (left) and local recurrence-free survival (right) for the subgroups (a) deep soft tissue angiosarcomas and (b) primary vs. secondary angiosarcomas showing hazard ratio (HR), 95\% confidence interval (CI), and $p$ value.

For local recurrence-free survival, the etiology 'secondary angiosarcoma' was identified as a negative prognostic factor in all subgroups, but was only significant for angiosarcomas of the breast (Supplement Figure 1). For metastasis-free survival, no significant prognostic factor by multivariate Cox regression analysis could be detected (Supplement Figure 2).

\section{Discussion}

In this retrospective single-center study, we evaluated the prognostic value of clinical factors with regard to overall survival as well as local recurrence-free and metastasis-free survival in patients with localized angiosarcoma. The rarity and heterogeneity of angiosarcomas pose barriers to comprehensive studies.

Our study is one of the largest single-center studies for localized angiosarcomas with 136 patients, compared to 28-324 patients in other studies, whereas not all focused on localized disease $[5,6,8,17-21]$. The median age in our cohort with 67 years and a slight dominance of female patients are comparable to other cohorts $[5,6,8,17-21]$. In contrast to other studies, we systematically focused on localized angiosarcomas and compared the main clinical subtypes, namely, cutaneous angiosarcomas, angiosarcomas of the breast, deep soft tissue angiosarcomas, and primary and radiation-associated angiosarcomas. We also included lymphedema-associated angiosarcomas in our analysis, but with only four patients, no statistical analyses could be performed. Overall, the distribution of angiosarcoma subtypes is comparable to other studies, although a high variability can be observed between studies: cutaneous angiosarcomas (13-75\%), angiosarcomas of the breast (16-56\%), deep soft tissue angiosarcomas (5-42\%), and primary (46-71\%), radiation-associated (17-63\%), and lymphedema-associated (3-22\%) angiosarcomas $[5,6,8,17-21]$. The overall survival for localized angiosarcomas varies in other studies with a median time from 20.8 to $>60$ months and 5-year survival rates from $17.9 \%$ to $74.8 \%$, whereas our cohort ranges somehow in the middle $[5,6,8,17-19,21]$. Local recurrence-free survival and metastasis-free survival were, in most studies, not systematically investigated. The 2-year local control rates are reported with $58 \%$ to $75 \%$, which are a bit higher compared to our cohort $[6,8,17,20]$. This difference might be explained by the fact that many patients in our cohort were not primarily resected at a specialized center and only referred subsequently to our sarcoma center. Median metastasis-free survival was reported lower in one study with 36.1 months compared to our cohort, and 2-year distant control rates were reported from $46.4 \%$ to $81.4 \%[6,8,20]$. This high variation is very likely due to the high heterogeneity of clinical subtypes summarized under the umbrella of angiosarcomas. Therefore, our study aimed to compare the subtypes of angiosarcoma and to evaluate prognostic factors.

While other studies reported a shorter median overall survival for cutaneous angiosarcomas (19.7-29 months), the 2 - and 5-year overall survival rates were comparable [22-25]. Here, the site of the cutaneous angiosarcomas seems important, with a worse prognosis for angiosarcomas of the scalp compared to the face/neck and outside the head/neck $[19,22,25]$. In our cohort, the majority was located at the head and neck, and due to our reporting system, discrimination between the scalp, face, and neck was not possible. Regarding local recurrence in cutaneous angiosarcomas, most studies reported a better 5-year local control rate between $18 \%$ and $43 \%$ [22, 23]. Metastasis-free survival was 
not systematically investigated in other studies [22-25]. For angiosarcomas of the breast, two meta-analyses with patient numbers of 222 [26] and 975 [27] and one retrospective study $(N=49)$ [28] presented comparable data for overall survival, with a 2-year overall survival rate of 71.1\% [28], a 5year overall survival rate of $43 \%$ [26], and a wide range for median overall survival from 12 to 72 months, with a comparable average to our cohort of around 43 months [27]. Other retrospective studies observed higher 5-year rates for overall survival $(61-69 \%)[29,30]$. The local control in other studies on angiosarcomas of the breast showed a better prognosis for 2 years with $55.2 \%, 5$ years from $44 \%$ to $62 \%$, and median recurrence-free survival from 6 to 54 months, averaging at 18 months [26-29]. Our cohort contained mainly radiation-associated angiosarcomas, which might explain the high risk of local recurrence. This was supported by the multivariate analyses, where secondary angiosarcoma was the only significant risk factor for local recurrence. Only limited data are published on metastasis, which is generally seen with low risk for angiosarcomas of the breast, with a reported 2-year metastasis-free survival rate of $57.3 \%$ and median time of 13.2 months [28, 29]. Regarding deep soft tissue angiosarcomas, to our knowledge, no study so far focused on the analysis of localized disease. However, these deep soft tissue angiosarcomas were included in studies about the prognosis of angiosarcomas in general or studies focused on certain organs, such as the liver, kidney, lung, or heart $[1,5,6,8,17]$. Here, median overall survival was reported similarly poor between 2.8 and 18.2 months $[5,6,8,21]$. One study also addressed local recurrence and metastasis, showing a lower local recurrence-free survival of 17 months and comparable poor metastasis-free survival of 11 months [8].

Our study demonstrates a similar behavior of cutaneous angiosarcomas and angiosarcomas of the breast with comparable median overall survival, local recurrence-free survival, and metastasis-free survival. Notably, the median metastasis-free survival was longer than the median overall survival, suggesting that a significant number of patients died of their local relapse. This is underscored by a very short median local recurrence-free survival for both subtypes, which is in accordance with other studies $[8,24,26]$. On the contrary, deep soft tissue angiosarcomas showed worse median overall survival, which was associated with reduced median metastasis-free survival time.

In our cohort, we also compared primary to secondary angiosarcomas, namely, radiation- and lymphedema-associated. In contrast to several other studies, we did not separate UV-associated angiosarcomas from primary angiosarcomas. The worst prognosis was seen for the four lymphedema-associated angiosarcomas, which is in accordance with previous reports $[5,8]$. For radiation-associated angiosarcomas, we observed a nonsignificant trend towards a better median overall survival and metastasis-free survival compared to primary angiosarcomas. The opposite was seen for local recurrence-free survival, which was significantly worse. Here, a clear tendency was also observed in the multivariate analysis, but only significant for secondary angiosarcomas of the breast. The results from other studies are diverse. While some do not observe a significant difference in overall survival $[18,31,32]$, other studies showed better or worse prognosis for primary vs. secondary angiosarcomas $[5,20]$. Two studies confirmed the worse prognosis of radiation-associated angiosarcomas in local recurrence (mLRFS: 20.1 months vs. 38 months; 2 y LC: $31.9 \%$ vs. $72.4 \%$ ) but showed contradicting results with worse prognosis as well in overall survival (mOS: 26.5 months vs. $39.9-45.9$ months; 2 y OS: $45 \%$ vs. $65 \%$ ) and distant free survival (mDFS: 26.1 months vs. 29-39 months; 2 y DC: $41.4 \%$ vs. $73.4 \%$ ) $[8,20]$.

To obtain prognostic factors, we performed univariate and multivariate analyses. We excluded tumor grade as a covariate as this is no longer considered applicable to angiosarcomas [33]. In univariate analyses, tumor size $>10 \mathrm{~cm}$, positive surgical margin, and positive or unknown lymph node status were significantly associated with worse overall survival in the cohort of all localized angiosarcomas. Hereby, tumor size $>10 \mathrm{~cm}$ was also significantly associated with shorter local recurrence-free and metastasis-free survival, but multivariate analyses did not confirm tumor size, surgical margin, and lymph node status as independent prognostic factors. This might be due to the high number of unknown tumor sizes and not evaluable surgical margins and lymph node status. With regard to size, estimation of tumor extent could be vastly underestimated in patients with any skin-associated angiosarcoma. For these patients, surgical margins may also be more difficult to determine as tumor cells grow rather diffuse and therefore leave more frequently cutaneous skip metastases. Previous studies have reported tumor size, mostly $>5 \mathrm{~cm}[6,8,24,26-28,30]$, and positive surgical margins as negative prognostic factors in angiosarcomas $[8,21,23]$. In line with previous studies, the only independent positive prognosis factor for overall survival, observed in all clinical subtypes, was age $<70$ years $[5,8,19,24,26]$.

Our study demonstrates a distinct different behavior of the subtypes with regard to local recurrence, metastasis, and overall survival. While deep soft tissue angiosarcomas showed a high risk of metastasis, in cutaneous angiosarcomas, angiosarcomas of the breast, and secondary angiosarcomas, the risk of local recurrence was increased. There are several limitations. Although the number of localized angiosarcomas is high and comparable to other studies, the numbers in the subgroups are too low to perform valid statistical analysis for all prognostic factors within the subgroups. Moreover, the clinical subgroups of cutaneous angiosarcomas, angiosarcomas of the breast, and deep soft tissue angiosarcomas are still quite heterogeneous. We did not consider the exact anatomic site or distinguish the etiology within each of the subgroups (cutaneous angiosarcoma, angiosarcoma of the breast, and deep soft tissue angiosarcoma). Moreover, for many patients, clinical information on tumor size, surgical margins, and lymph node status was, due to the retrospective analysis, not determinable.

Nevertheless, our study provides several implications for consulting patients, therapy planning, and future clinical studies. Our data indicate a high risk of local recurrence and 
lower risk for early metastasis in cutaneous angiosarcomas and angiosarcomas of the breast, whereas for the latter, our data are predominantly based on secondary angiosarcomas. These are rather angiosarcomas of the chest wall and behave clinically different to primary angiosarcomas of the breast. As discussed above, local tumor control may have a major impact on survival. However, this is hampered by the fact that cutaneous and radiation- and lymphedema-associated angiosarcomas are not contained within a compartment, but often show blurry margins, and neither inspection nor imaging allows to exactly determine the extent of the disease. Due to this, the tumor size often cannot be determined, and TNM classification for the primary site seems obsolete. Here, improved methods for local staging have to be investigated and implemented. In addition to the difficulty to determine the exact tumor expansion, often, limited perioperative therapy options are available, especially in radiation-associated angiosarcomas, due to pretreatment for initial malignant disease. Radical surgical resection represents, by far, the most important therapeutic modality to cure these tumors. For radiation-associated angiosarcomas of the chest wall, radical excision that includes the complete irradiated field followed by chest wall reconstruction had a significant positive impact on local control, distant metastasis, and disease-specific survival $[34,35]$. Such radical resection is technically impossible in many patients with cutaneous angiosarcomas of the head and neck and lymphedema-associated angiosarcomas. The role of multimodal approaches, such as hyperthermic isolated limb perfusion with TNFalpha and melphalan [36] or perioperative chemo- and radiotherapy, is yet disputed, but many centers consider it as part of primary treatment in younger patients with good performance status $[8,14,15,27,36]$. For deep soft tissue angiosarcomas, distant disease control seems to be particularly important. Recently, Pasquali et al. demonstrated a better survival for patients with low predicted overall survival when adjuvant chemotherapy was applied, which is true for most angiosarcomas [37]. Unfortunately, retrospective analyses from randomized trials do not allow conclusions due to the low numbers of angiosarcoma patients that were included. Another topic is recommendations for follow-up after curative treatment which are not standardized, and evidence-based data are missing. Interestingly, the Kaplan-Meier plots for overall survival, local recurrence-free survival, and metastasis-free survival in our cohort show a plateau between 5 and 6 years for all clinical subtypes, which is similar to other studies $[5,6,8,20,21]$. This suggests that follow-up in angiosarcomas should be planned for a minimum of 5 to rather 6 years.

\section{Conclusion}

Angiosarcomas represent a heterogeneous sarcoma subtype with strikingly different clinical behaviors and prognoses depending on the primary site and etiology. Local control should be the focus for cutaneous angiosarcomas, especially of the head/neck, and radiation-associated angiosarcomas as local relapses define the outcome for most patients.
Therapeutic concepts should incorporate early and aggressive surgical and, wherever possible, radiotherapeutic treatment. Given the very high risk of the metastatic disease in deep soft tissue angiosarcomas, long-term survival will only improve with the use of systemic treatments. The lack of prospective and randomized trials for this subgroup of patients poses a considerable challenge for advising patients.

$\begin{array}{ll}\text { Abbreviations } \\ \text { AS: } & \text { Angiosarcoma } \\ \text { CI: } & \text { Confidence interval } \\ \text { cm: } & \text { Centimeters } \\ \text { DC: } & \text { Distant control } \\ \text { mDFS: } & \text { Median distant free survival } \\ \text { HR: } & \text { Hazard ratio } \\ \text { LC: } & \text { Local control } \\ \text { LRFS: } & \text { Local recurrence-free survival } \\ \text { MFS: } & \text { Metastasis-free survival } \\ \text { mLRFS: } & \text { Median local recurrence-free survival } \\ \text { mMFS: } & \text { Median metastasis-free survival } \\ \text { mOS: } & \text { Median overall survival } \\ \text { N: } & \text { Number } \\ \text { n.a.: } & \text { Not applicable } \\ \text { OS: } & \text { Overall survival } \\ \text { OP: } & \text { Operation } \\ \text { p: } & \text { p value } \\ \text { PF: } & \text { Prognostic factor } \\ \text { Tx: } & \text { Therapy } \\ \text { UV: } & \text { Ultraviolet } \\ \text { vs.: } & \text { Versus } \\ \text { y: } & \text { Years. } \\ & \end{array}$

\section{Data Availability}

The datasets used and/or analyzed during the present study are available from the corresponding author upon reasonable request.

\section{Ethical Approval}

Ethical approval was obtained from the local certified Ethics Committee of the Medical Faculty, University of DuisburgEssen, Essen, Germany (approval no. 21-9825-BO).

\section{Consent}

Consent was not obtained from individual patients. However, all patient information was anonymized by the investigators for analysis.

\section{Conflicts of Interest}

LEP reports consultation for Belpharma outside of the submitted work. CP reports honoraria from Roche, Boehringer Ingelheim, and AstraZeneca outside the submitted work. JTS reports personal fees from AstraZeneca, Aurikamed, Bayer, Baxalta, BMS, Celgene, Falk Foundation, Immunocore, iOMEDICO, Lilly, Novartis, Roche, and Shire; grants and personal fees from Bristol Myers Squibb, Celgene, 
and Roche; has minor equity in iTheranostics and Pharma15 $(<3 \%)$; and is a member of the Board of Directors for Pharma15, all outside the submitted work. SB reports personal fees from Bayer, Eli Lilly, Novartis, Pfizer, and PharmaMar; serves in an advisory/consultancy role for ADC Therapeutics, Bayer, Blueprint Medicines, Daiichi Sankyo, Deciphera, Eli Lilly, Exelixis, Janssen-Cilag, Nanobiotix, Novartis, PharmaMar, Plexxikon, and Roche; receives research funding from Novartis; and serves as a member of the External Advisory Board of the Federal Ministry of Health for "Off-label use in oncology," all outside the submitted work. RH reports travel grants from Lilly, Novartis, and PharmaMar, as well as personal fees from Lilly outside the submitted work. All other authors declare no conflicts of interest concerning this specific publication.

\section{Authors' Contributions}

IS, SB, and RH conceived the present study. IS, FF, MA, and FZM collected the data. IS and RH collected the data and reviewed the cases for the localized disease. IS, STL, and RH planned the statistical analysis. IS and STL performed statistical analysis. IS and RH wrote the manuscript. IS, STL, FF, JF, HUST, JWT, LEP, CP, HUS, MA, UD, JTS, SB, and $\mathrm{RH}$ interpreted the data and revised the manuscript for the intellectual content. All authors read and approved the manuscript.

\section{Acknowledgments}

RH was supported by the Clinician Scientist Program of the University Medicine Essen Clinician Scientist Academy (UMEA) sponsored by the Faculty of Medicine and Deutsche Forschungsgemeinschaft (DFG).

\section{Supplementary Materials}

Supplement Figure 1: multivariate analysis. Forest plots of multivariate Cox regression analysis on overall survival (left) and local recurrence-free survival (right) for the subgroups A) cutaneous angiosarcomas and B) angiosarcomas of the breast showing hazard ratio (HR), 95\% confidence interval $(\mathrm{CI})$, and $p$ value. Supplement Figure 2: multivariate analysis of metastasis-free survival. Forest plots of multivariate Cox regression analysis on metastasis-free survival for the subgroups A) cutaneous angiosarcomas, B) angiosarcomas of the breast, C) deep soft tissue angiosarcomas, and D) primary vs. secondary angiosarcomas showing hazard ratio (HR), 95\% confidence interval (CI), and $p$ value. (Supplementary Materials)

\section{References}

[1] R. J. Young, N. J. Brown, M. W. Reed, D. Hughes, and P. J. Woll, "Angiosarcoma," The Lancet Oncology, vol. 11, no. 10, pp. 983-991, 2010.

[2] G. De Pinieux, M. Karanian, F. Le Loarer et al., "Nationwide incidence of sarcomas and connective tissue tumors of intermediate malignancy over four years using an expert pathology review network," PLoS One, vol. 16, no. 2, Article ID e0246958, 2021.

[3] M. Dennis NF and G. Lawrence, "Soft tissue sarcoma incidence and survival tumours diagnosed in england between 1985 and 2009," 2012, http://wwwncinorguk/cancer_type_and_topic_ specific\%20work/cancer_type_specific_work/sarcomas/.

[4] A. Trama, G. Badalamenti, G. G. Baldi et al., "Soft tissue sarcoma in Italy: from epidemiological data to clinical networking to improve patient care and outcomes," Cancer Epidemiology, vol. 59, pp. 258-264, 2019.

[5] M. E. Weidema, U. E. Flucke, W. T. A. Van der Graaf et al., "Prognostic factors in a large nationwide cohort of histologically confirmed primary and secondary angiosarcomas," Cancers, vol. 11, no. 11, 2019.

[6] D. Buehler, S. R. Rice, J. S. Moody et al., "Angiosarcoma outcomes and prognostic factors," American Journal of Clinical Oncology, vol. 37, no. 5, pp. 473-479, 2014.

[7] V. Florou and B. A. Wilky, "Current and future directions for angiosarcoma therapy," Current Treatment Options in Oncology, vol. 19, no. 3, p. 14, 2018.

[8] C. Lindet, A. Neuville, N. Penel et al., "Localised angiosarcomas: the identification of prognostic factors and analysis of treatment impact. A retrospective analysis from the French Sarcoma Group (GSF/GETO)," European Journal of Cancer, vol. 49, no. 2, pp. 369-376, 2013.

[9] V. Florou, A. E. Rosenberg, E. Wieder et al., "Angiosarcoma patients treated with immune checkpoint inhibitors: a case series of seven patients from a single institution," Journal for ImmunoTherapy of Cancer, vol. 7, no. 1, p. 213, 2019.

[10] R. Hamacher, D. Kämpfe, K. Reuter-Jessen et al., "Dramatic response of a PD-L1-positive advanced angiosarcoma of the scalp to pembrolizumab," JCO Precision Oncology, vol. 20, pp. 1-7, 2018.

[11] C. A. Painter, E. Jain, B. N. Tomson et al., "The Angiosarcoma Project: enabling genomic and clinical discoveries in a rare cancer through patient-partnered research," Nature Medicine, vol. 26, no. 2, pp. 181-187, 2020.

[12] A. M. Udager, M. K. Ishikawa, D. R. Lucas, J. B. McHugh, and R. M. Patel, "MYC immunohistochemistry in angiosarcoma and atypical vascular lesions: practical considerations based on a single institutional experience," Pathology, vol. 48, no. 7 , pp. 697-704, 2016.

[13] E. Esposito, F. Avino, R. di Giacomo et al., "Angiosarcoma of the breast, the unknown-a review of the current literature," Translational Cancer Research, vol. 8, no. 5, pp. S510-S517, 2019.

[14] K. T. Lee, J. Moon, H. S. Jeong, H. S. Lim, and S. Y. Lim, "Benefits of the multidisciplinary approach after curative surgery for the treatment of scalp angiosarcoma," Annals of Plastic Surgery, vol. 44, 2020.

[15] J. Oxenberg, N. I. Khushalani, K. E. Salerno, K. Attwood, and J. M. Kane, "Neoadjuvant chemotherapy for primary cutaneous/soft tissue angiosarcoma: determining tumor behavior prior to surgical resection," Journal of Surgical Oncology, vol. 111, no. 7, pp. 829-833, 2015.

[16] A. J. M. Rombouts, J. Huising, N. Hugen et al., “Assessment of radiotherapy-associated angiosarcoma after breast cancer treatment in a Dutch population-based study," JAMA Oncology, vol. 5, no. 2, pp. 267-269, 2019.

[17] J. Fayette, E. Martin, S. Piperno-Neumann et al., "Angiosarcomas, a heterogeneous group of sarcomas with specific behavior depending on primary site: a retrospective study of 161 cases," Annals of Oncology, vol. 18, no. 12, pp. 2030-2036, 2007. 
[18] G. Lahat, A. R. Dhuka, H. Hallevi et al., "Angiosarcoma," Annals of Surgery, vol. 251, no. 6, pp. 1098-1106, 2010.

[19] B. L. Lee, C. F. Chen, P. C. Chen et al., "Investigation of prognostic features in primary cutaneous and soft tissue angiosarcoma after surgical resection: a retrospective study," Annals of Plastic Surgery, vol. 78, no. 3, pp. S41-S6, 2017.

[20] E. Merfeld, P. Gabani, M. B. Spraker et al., "Clinical outcomes and prognostic features of angiosarcoma: significance of prior radiation therapy," Clinical Oncology, vol. 31, no. 4, pp. 232-241, 2019.

[21] A. Smrke, J. Hamm, A. Karvat, C. Simmons, and A. Srikanthan, "A retrospective review of 145 patients with angiosarcoma: radiation therapy, extent of resection and chemotherapy are important predictors of survival," Molecular and Clinical Oncology, vol. 13, no. 2, pp. 179-185, 2020.

[22] J. M. Bernstein, J. C. Irish, D. H. Brown et al., "Survival outcomes for cutaneous angiosarcoma of the scalp versus face," Head \& Neck, vol. 39, no. 6, pp. 1205-1211, 2017.

[23] C. H. Kuan, H. W. Yang, H. F. Huang et al., "Prognostic significance of positive surgical margins for scalp angiosarcoma," Journal of the Formosan Medical Association, vol. 32, 2020.

[24] K. C. Lee, S.-K. Chuang, E. M. Philipone, and S. M. Peters, "Characteristics and prognosis of primary head and neck angiosarcomas: a surveillance, epidemiology, and end results program (seer) analysis of 1250 cases," Head and Neck Pathology, vol. 13, no. 3, pp. 378-385, 2019.

[25] I. J. Moon, Y. J. Kim, C. H. Won et al., "Clinicopathological and survival analyses of primary cutaneous angiosarcoma in an Asian population: prognostic value of the clinical features of skin lesions," International Journal of Dermatology, vol. 59, no. 5, pp. 582-589, 2020.

[26] A. L. Depla, C. H. Scharloo-Karels, M. A. A. de Jong et al., "Treatment and prognostic factors of radiation-associated angiosarcoma (RAAS) after primary breast cancer: a systematic review," European Journal of Cancer, vol. 50, no. 10, pp. 1779-1788, 2014.

[27] Y. Abdou, A. Elkhanany, K. Attwood, W. Ji, K. Takabe, and M. Opyrchal, "Primary and secondary breast angiosarcoma: single center report and a meta-analysis," Breast Cancer Research and Treatment, vol. 178, no. 3, pp. 523-533, 2019.

[28] R. B. Cohen-Hallaleh, H. G. Smith, R. C. Smith et al., "Radiation induced angiosarcoma of the breast: outcomes from a retrospective case series," Clinical Sarcoma Research, vol. 7, no. 1, p. 15, 2017.

[29] S. H. Salminen, T. Wiklund, M. M. Sampo et al., "Treatment and prognosis of radiation-associated breast angiosarcoma in a nationwide population," Annals of Surgical Oncology, vol. 27, no. 4, pp. 1002-1010, 2020.

[30] T. Sher, B. T. Hennessy, V. Valero et al., "Primary angiosarcomas of the breast," Cancer, vol. 110, no. 1, pp. 173-178, 2007.

[31] A. J. Sinnamon, M. G. Neuwirth, M. T. McMillan et al., “A prognostic model for resectable soft tissue and cutaneous angiosarcoma," Journal of Surgical Oncology, vol. 114, no. 5, pp. 557-563, 2016.

[32] L. Wang, I. W. Lao, L. Yu, and J. Wang, "Clinicopathological features and prognostic factors in angiosarcoma: a retrospective analysis of 200 patients from a single Chinese medical institute," Oncology letters, vol. 14, no. 5, pp. 5370-5378, 2017.

[33] A. T. Deyrup and S. W. Weiss, "Grading of soft tissue sarcomas: the challenge of providing precise information in an imprecise world," Histopathology, vol. 48, no. 1, pp. 42-50, 2006.
[34] L. Feinberg, A. Srinivasan, J. K. Singh et al., "Impact of specialist management on survival from radiation-associated angiosarcoma of the breast," British Journal of Surgery, vol. 105, no. 4, pp. 401-409, 2018.

[35] G. Z. Li, M. Fairweather, J. Wang, D. P. Orgill, M. M. Bertagnolli, and C. P. Raut, "Cutaneous radiationassociated breast angiosarcoma," Annals of Surgery, vol. 265, no. 4, pp. 814-820, 2017.

[36] F. Farzaliyev, R. Hamacher, H. U. Steinau Professor, S. Bertram, and L. E. Podleska, "Secondary angiosarcoma: a fatal complication of chronic lymphedema," Journal of Surgical Oncology, vol. 121, no. 1, pp. 85-90, 2020.

[37] S. Pasquali, S. Pizzamiglio, N. Touati et al., "The impact of chemotherapy on survival of patients with extremity and trunk wall soft tissue sarcoma: revisiting the results of the EORTC-STBSG 62931 randomised trial," European Journal of Cancer, vol. 109, pp. 51-60, 2019. 